\title{
Video Frame Illumination Inconsistency Reduction using CLAHE with Kekre's LUV Color Space
}

\author{
Deepa Abin, Sudeep D. Thepade
}

\begin{abstract}
Visual frame quality is of utmost significance and is relevant in numerous computer vision applications such as object detection, video surveillance, optical motion capture, multimedia and human computer interface. Under controlled or uncontrolled environment, the video visual frame quality gets affected due to illumination variations. This may further hamper the interpretability and may lead to significant loss of information for background modeling. An excellent background model can enhance good visual perception. In this work, local enhancement technique with improved background modeling, Clipped Adaptive Histogram Equalization (CLAHE) is explored with Kekre's LUV color space to reduce the illumination inconsistency especially with darker set of video frames and a significant improved average entropy of 7.7225 has been obtained, which is higher than the existing explored variations of CLAHE. .

Keywords: Illumination Inconsistency, Background Modeling, Clipped Adaptive Histogram Equalization, Kekre's LUV color space.
\end{abstract}

\section{INTRODUCTION}

In the field of digital videos, the quality of visual frame is very crucial. Non uniform illumination is one of the major factors hampering the quality of video frame [1]-[3]. Illumination change can occur due to climate changes, sudden alterations of light etc. Inconsistent illumination can affect several applications in the areas of medical diagnosis using imaging, remote sensed imaging, aerial imaging and underground imaging [4]-[10]. There are occurrences of sudden on-off variations in light or sometimes those can be even gradual. There is a need to swiftly adapt to such illumination inconsistencies [2]-[5],[9].

Illumination inconsistency can be present in the overall video frame or in the foreground and also in the background. The irregular illumination in video frames have to be regularized for the visual enhancement of finer details [1]-[7].

In the recent time, there has been significant contribution by researchers to address the background modeling challenges [1]-[8]. Acquiring a background frame that does not include any illumination inconsistency is very crucial. There has been

\footnotetext{
Revised Manuscript Received on February 05, 2020.

* Correspondence Author

Deepa Abin*, Computer Department, PCCoE-SPPU,Pune,India deepaabin@gmail.com

Sudeep D. Thepade, Computer Department, PCCoE-SPPU,Pune,India. sudeepthepade@gmail.com
}

(C) The Authors. Published by Blue Eyes Intelligence Engineering and Sciences Publication (BEIESP). This is an open access article under the CC BY-NC-ND license (http://creativecommons.org/licenses/by-nc-nd/4.0/) a breakthrough with computer vision to design systems that address the light variations in outdoor scenes in dynamic environment, however a background modeling method robust enough to handle illumination inconsistencies is needed. There is evergrowing requirement to devise the methods for interpretation of video frames for better understanding of the perceptions; this makes it absolutely essential to have the video frames free from irregularities like noise or uneven illumination [1]-[9]. Therefore there is a dire need to build a robust and adaptive model for both controlled and uncontrolled environment [3]-[5].

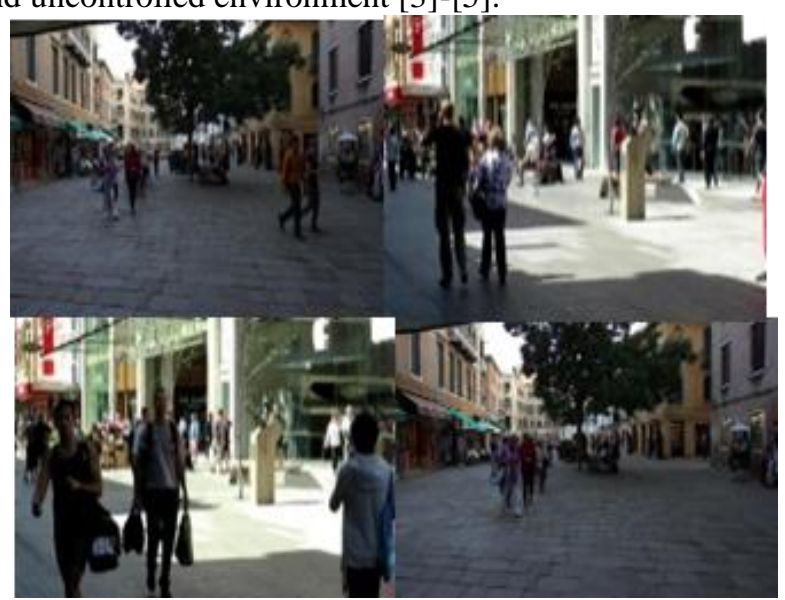

Fig.1. Glimpse of Illumination Inconsistent Video Frames As seen in Fig.1, a glimpse of illumination inconsistent video frames is presented. Through this work an exploration of local enhancement techniques has been carried out for such video frame illumination inconsistency reduction. The further paper is organized as in the Section II Literature Survey is presented, in Section III Proposed Method is discussed, Section IV focuses on Results and Discussion followed by Section $\mathrm{V}$ that summarizes the Concluding Remarks.

\section{LITERATURE SURVEY}

Illumination variations in the video frames needs to be robustly handled through Background Modeling methods. In literature several approaches exist addressing the area of correcting inconsistent illumination. In digital image processing, Histogram Equalization (HE) [10]-[20] has been a simple prevalent method for contrast improvement in images. It uses cumulative density function to obtain the result. Fundamentally, the HE technique can be categorized in mainly classes such as Global enhancement methods and Local enhancement methods. 
The GHE (Global Histogram Equalization) methods, globally updates the image histogram. These procedures are simpler for implementation

and delivers substantial visual perceptibility, however with contrast loss for few sections in images at times. In particular frames, local areas are left unenhanced in case of global enhancement methods. Over enhancement and significant contrast loss for image regions is seen in case of global enhancement methods. Ketcham [17] putforth the limitation of global histogram equalization that it is inadequate for enhancement of minor local details and also further suggested "LAHE" (Local Area Histogram Equalization). It is also known as AHE (Adaptive Histogram Equalization). The "LHE" (Local Histogram Equalization) methods are put forth as in Fig.2.

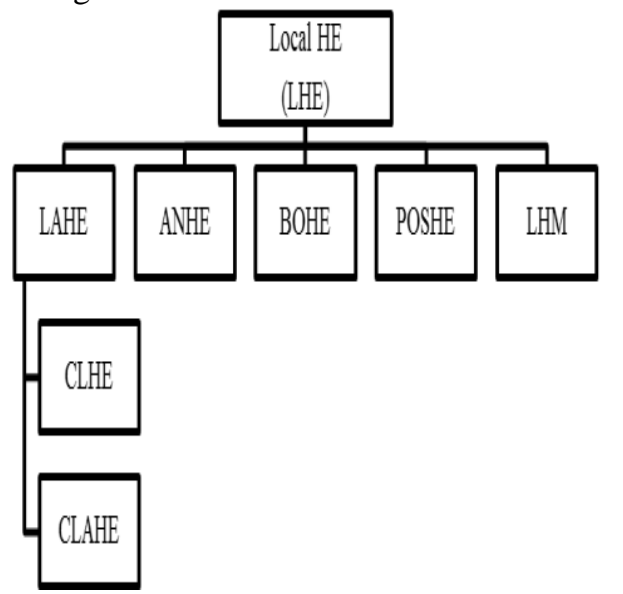

Fig.2. Local Histogram Equalization Methods

In “AHE” [9],[15]-[17] a contextual region around a center pixel is defined using a small window. Calculation of the $\mathrm{CDF}$ is based on this contextual region pixels. The data of neighbour pixels in contextual region is used for transform function in LHE. It makes good use of local information. It depicts the full dynamic intensity range as required in many display devices. However the processing time is more and there is a problem of over enhancement seen in few cases. The local methods can also be broadly categorized as LAHE [12],[15]-[18], “LHM" (Local Histogram Matching) [12],[16]-[19] and "LNORM" ( Local Normal Distribution) [12],[16]-[18]. LAHE is further extended to "CLHE" (Contrast Limited Histogram Equalization). This got eventually extended to "CLAHE" (Contrast Limited Adaptive Histogram Equalization )..In [21]-[24], the CLHE is based on histogram chopping at specific threshold values to further equalize to improve frame's local contrast. The over enhancement can be avoided by clipping the histogram peak in each tile. Certain concepts of AHE and CLHE are used in CLAHE (Contrast Limited Adaptive Histogram Equalization). The noise amplification is limited in CLAHE, hence more natural output is obtained. It is highly useful in medical imaging and video broadcasting where the brightness requirement is vital.The hidden features of particular frames can be visible with the brightness level enhancement. Easier calculation, good outputs and simplicity are vital features of CLAHE. However in few cases, time consumption can be more.

In "ANHE" (Adaptive Neighbourhood Histogram Equalization) [12],[15]-[18], a window with no particular dimensions or shape is used for the histogram computation for the region. ANHE is additionally adaptive method for contrast enhancement as compared to LAHE, as the edge as well as window artifacts present in outcome of LAHE, are overcome by ANHE. In "BOHE" (Block Overlapped HE) [12],[16]-[18] method, HE is peformed on the histogram of rectangular sub block of the frame. Then, HE is performed on the center pixel and later this center is moved to the adjacent pixel and the process is repeated to obtain uniformly illuminated regions. The computational complexity is however high. In "POSHE" (Partially Overlapped Sub-Block HE) [12],[17]-[19] computation complexity is reduced as there is no over lapping of sub blocks on which HE is applied. Shape differences might appear in this process which can be overcome using weighted sum of histograms obtained from neighbouring sub blocks. "Local Normal Distribution" [12],[16]-[18], are similar to their global counterparts but are applied locally for noise removal. Solving uneven lighting issues in frames and a mathematical model inception for the same is indeed a big challenge. The illumination inconsistencies in a video frame once reduced can be further applicable for real life applications as subjectively and objectively perception would be improved.

\section{PROPOSED METHOD}

As per the literature, Local enhancement algorithm, CLAHE [21]-[24] has better prospect and thus in earlier work CLAHE is explored with the different color spaces such as CLAHE_Lab [21],[22] and CLAHE_YCbCr [23]. The CLAHE has been further extended in this work with Kekre's LUV [25],[26]color space and referred as CLAHE_LUV.

Here in Kekre's LUV color model, L gives luminance and U, $\mathrm{V}$ gives chromaticity values of color video frame. Negative value of $U$ indicates prominence of red component in color image and negative value of $\mathrm{V}$ indicates prominence of green component over blue. The novel extension with Kekre's LUV color space is implemented with CLAHE for subjective and objective evaluation. The results obtained are significantly improved with reference to earlier explorations CLAHE_Lab [21],[22] and CLAHE_YCbCr [23].The flowchart as in Fig.3, states the methodology used for the extension of CLAHE with Kekre's LUV color space. As seen in Fig.3, the input video frame is in RGB color space with inconsistent illumination. Here the 'KSD color space' is a generic luminance-chromaticity color space model considered where $\mathrm{K}$ is the luminance component and S,D are the chromaticity components. These S,D chromaticity components in Lab color space represent $\mathrm{a}$, b and in $\mathrm{YCbCr}$ color space the chromaticity components are $\mathrm{Cb}$ and $\mathrm{Cr}$. In proposed work of CLAHE with Kekre's LUV color space, the chromaticity components are $\mathrm{U}$ and $\mathrm{V}$. The clipped regions of luminance plane ' $\mathrm{K}$ ' are distributed with uniformity in CLAHE. The major limitation of global enhancement methods being unable to address all regions uniformly is very well overcome with CLAHE using Kekre's LUV color space. 


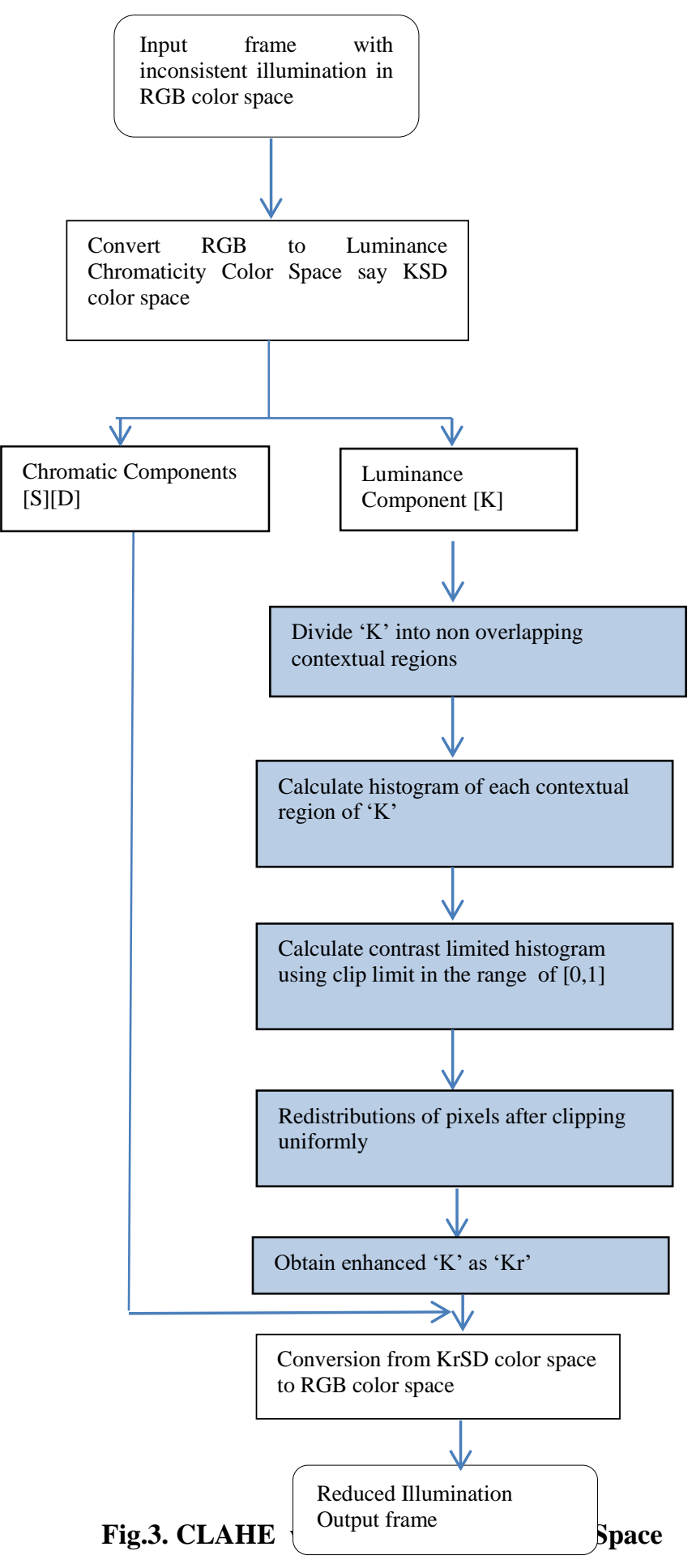

\section{RESULTS AND DISCUSSION}

In literature, the luminance chromaticity color spaces CIE-Lab and YCbCr explored with CLAHE as CLAHE_Lab [21],[22] and CLAHE_YCbCr [23]. The testbed used for proposed work are 100 sample frames obtained from one of the 21 video sequences in MOT17 dataset. The selective frames have been putforth as in Fig.4. The enhanced output frames as shown reflect better visual perception with reduced illumination inconsistency.
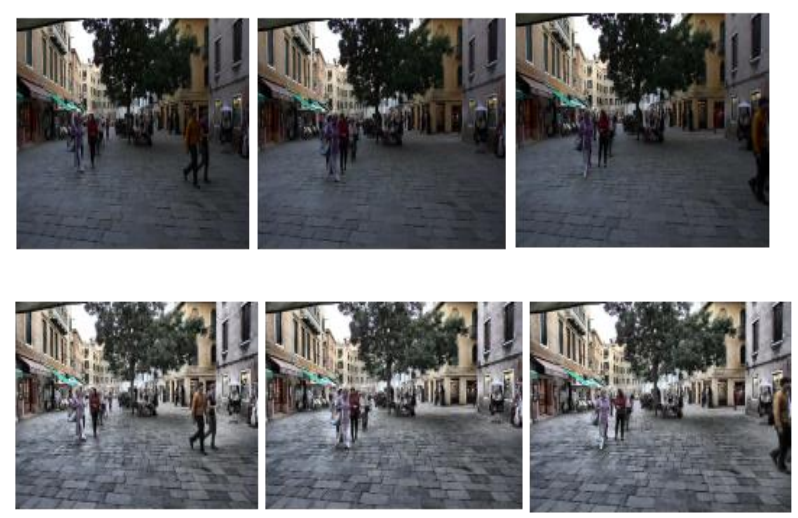

Fig.4. Selective Frames 1,2,3 Input and Enhanced Output Frames with CLAHE_LUV color spaceThe performance metric used to measure the quality enhancement here is the average entropy [27]-[30] value for the sample frames. Entropy measure is the No reference Image Quality assessment metric and higher the value, the richness of details is much more.Here Fig.5 illustrates the visual representation of performance metric, entropy measure for selected frames and the entropy value for selected frames with CLAHE_LUV has shown better improvement.

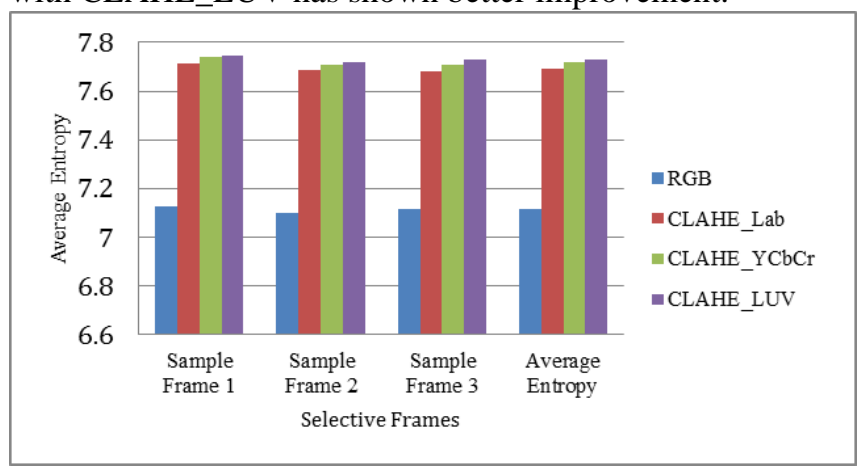

Fig.5.:Sample Entropy of Video Frames RGB to CLAHE_Lab, CLAHE_YCbCr and CLAHE_LUV

Table-II: Sample Entropy of Video Frames RGB to CLAHE_Lab, CLAHE_YCbCr, CLAHE_LUV

\begin{tabular}{|l|c|c|c|c|}
\hline Methods & $\begin{array}{l}\text { Sample } \\
\text { Frame 1 }\end{array}$ & $\begin{array}{l}\text { Sample } \\
\text { Frame 2 }\end{array}$ & $\begin{array}{l}\text { Sample } \\
\text { Frame 3 }\end{array}$ & $\begin{array}{l}\text { Average } \\
\text { Entropy }\end{array}$ \\
\hline RGB & 7.1277 & 7.1012 & 7.1169 & 7.1153 \\
\hline $\begin{array}{l}\text { CLAHE_Lab } \\
\text { [22] }\end{array}$ & 7.7119 & 7.6852 & 7.6832 & 7.6935 \\
\hline $\begin{array}{l}\text { CLAHE_YCbCr } \\
\text { [23] }\end{array}$ & 7.7382 & 7.7092 & 7.7075 & 7.7183 \\
\hline CLAHE_LUV & 7.747 & 7.7186 & 7.7288 & 7.7315 \\
\hline
\end{tabular}

The Table II, putforth further illustrates the entropy values for selective frames with existing illumination inconsistency reduction methods RGB, CLAHE_Lab[21,22], CLAHE_YCbCr[23] and also the proposed method CLAHE_LUV. Further as seen in Table III, the average entropy each for the input RGB method, CLAHE_Lab, CLAHE_YCbCr and CLAHE_LUV color space is putforth. Here as seen CLAHE_LUV has shown significant improvement with 7.225 over CLAHE_Lab and CLAHE_YCbCr color space.

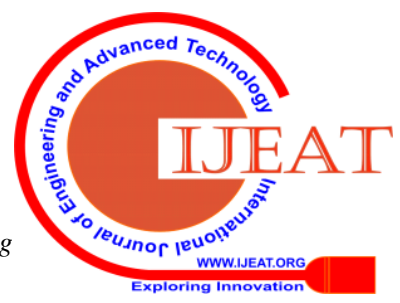


Table-III: Average Entropy of 100 sample frames with RGB, CLAHE_Lab, CLAHE_YCbCr and CLAHE_LUV

\begin{tabular}{|c|l|l|l|}
\hline Input & \multicolumn{3}{|c|}{ Enhanced Output } \\
\hline RGB & CLAHE_Lab & CLAHE_YCbCr & Proposed \\
{$[21]$} & {$[21],[22]$} & {$[23]$} & CLAHE_LUV \\
& & & \\
\hline 7.1012 & 7.6412 & 7.691 & 7.7225 \\
\hline
\end{tabular}

The graphical visualization of the average entropy on the testbed of 100 sample frames is putforth in Fig.6.

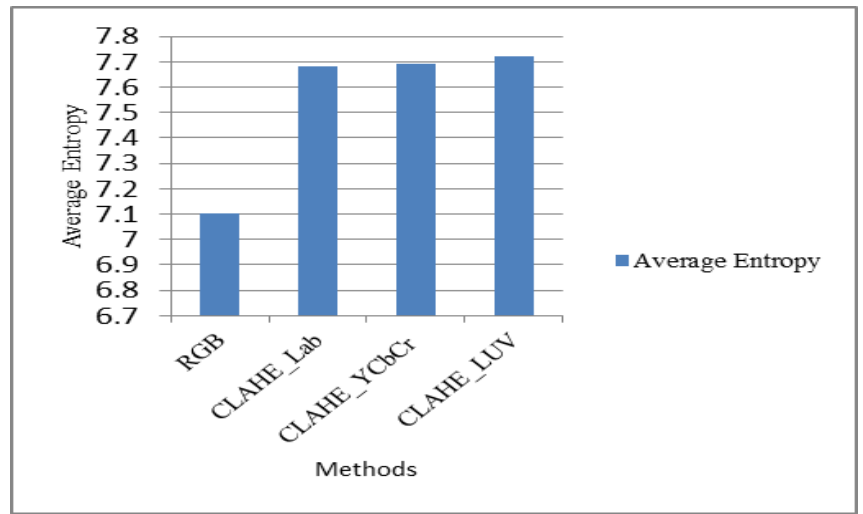

Fig.6.Average Entropy of Video Frames RGB ,CLAHE_Lab, CLAHE_YCbCr and proposed CLAHE_LUV

Through this work, significant results are achieved with Local Enhancement method, CLAHE. There is good scope for the exploration of fusion of local enhancement methods with global enhancement methods for improved background modeling with more consistent illumination.

\section{CONCLUSION}

The visual perceptual quality that gets hampered due to the inconsistent illumination and leads to significant loss of information for background modeling needs to be overcome. The improved visual quality of the frame can empower subjective perception and recognition tasks. Many techniques have been proposed for video frame quality enhancement with improved background modeling for reducing the inconsistent illumination. CLAHE is a good HE method comparatively among the local contrast enhancement methods and in this work, CLAHE_LUV has shown significant results for the illumination inconsistency especially with darker set of images with an average entropy of 7.7225; which is better than existing methods RGB, CLAHE_Lab and CLAHE_YCbCr.

There is ample amount of scope where the local contrast enhancement techniques can be fused and novel methodologies be developed to overcome the grey areas of both, for enhanced visual perception and illumination inconsistency reduction.

\section{REFERENCES}

1. M. Yazdi and T Bouwmans, "New trends on moving object detection in video images captured by a moving camera: A survey," Computer Science Review, vol. 28, 2018, pp. 157-177.
2. W Kim and C Jung, "Illumination-invariant background subtraction: Comparative review, models, and prospects", IEEE Access, vol. 5, 2017, pp. 8369-8384.

3. N.C Joy,J.C Prasad, N.C Joy and J. C Prasad, "Survey on background modeling and foreground detection methods," International Journal for Innovative Research in Science and Technology, vol. 3, 2016, pp. 338-41.

4. S. Jeeva and M. Sivabalakrishnan, "Survey on Background Modeling and Foreground Detection for Real Time Video Surveillance," Procedia Computer Science, vol. 50, 2015, pp. 566-571.

5. T. Bouwmans, F. El Baf and B. Vachon, "Statistical Background Modeling for Foreground Detection: A Survey," In Handbook of Pattern Recognition and Computer Vision, 2010, pp. 181-199.

6. T. Bouwmans, "Recent advanced statistical background modeling for foreground detection-a systematic survey," Recent Patents on Computer Science, vol. 4, 2011,147-176.

7. H. Hassanpour, M. Sedighi, and A.R. Manashty, "Video frame's background modeling: Reviewing the techniques," Journal of Signal and Information Processing, vol. 2, 2011, p.72.

8. Y Xu, J. Dong, B. Zhang, B, and D Xu, "Background modeling methods in video analysis: A review and comparative evaluation," CAAI Transactions on Intelligence Technology, vol. 1, 2016, pp. 43-60.

9. R.C. Gonzalez and R.E. Woods, Digital Image Processing, Third Edition, Pearson Prentice Hall, New Jersey, 2008.

10. N. Dey, "Uneven illumination correction of digital images: A survey of the state-of-the-art," Optik, vol. 183, 2019, pp. 483-495.

11. Dibya Jyoti Bora, "Importance of Image Enhancement Techniques in Color Image Segmentation: A Comprehensive and Comparative Study,” Indian J.Sci.Res, vol. 15, 2017, pp. 115-131.

12. S.D. Chen and A.R. Ramli,"Preserving brightness in histogram equalization based contrast enhancement techniques," Digital Signal Processing, vol. 14, 2004, pp. 413-428.

13. C.R. Nithyananda and A.C Ramachandra,"Survey on Histogram Equalization Method based Image Enhancement Techniques,” In 2016 IEEE International Conference on Data Mining and Advanced Computing (SAPIENCE), March 2016, pp. 150-158,

14. S. Rahman, M.M. Rahman, K. Hussain, S.M. Khaled and M. Shoyaib M, "Image Enhancement in Spatial Domain: A Comprehensive Study,'In 2014 17th IEEE International Conference on Computer and Information Technology (ICCIT), December 2014, pp. 368-373.

15. N.S.P. Kong, H. Ibrahim H and S.C Hoo, "A literature review on histogram equalization and its variations for digital image enhancement," International Journal of Innovation, Management and Technology, vol. 4, 2013, p. 386.

16. H. Wang and Y. Wang, "Recognizing face images under different lighting conditions", In 2003 IEEE International Conference on Acoustics, Speech, and Signal Processing (ICASSP'03), vol. 3, pp.131-141.

17. D.J. Ketcham, R.W. Lowe and J.W.Weber, Image enhancement techniques for cockpit displays (No. HAC-P74-530R),Hughes Aircraft Co Culver City Ca Display Systems Lab, 1974.

18. M. Kaur, J. Kaur, "Survey of contrast enhancement techniques based on histogram equalization," International Journal of Advanced Computer Science and Applications, 2011, p.2.

19. T.K. Kim, J.K. Paik and B.S. Kang, "Contrast enhancement system using spatially adaptive histogram equalization with temporal filtering," IEEE Transactions on Consumer Electronics, vol. 44, 1998, pp. 82-87.

20. S.S. Chiroliya and P. Mishra, Performance Analysis of Contrast Enhancement of Greyscale Images using Improved Histogram Equalization.

21. R. Kumar Rai, P. Gour and B.Singh, "Underwater Image Segmentation using CLAHE Enhancement and Thresholding," International Journal of Emerging Technology and Advanced Engineering, vol. 2, 2012, pp.118-123.

22. A. Alam, M. Abdullah and R.S. Mishra, "Colour Contrast Enhancement Method by Scaling the DC Coefficients in CIE-LAB Colour Space," International Journal of Computer Applications, vol. 97, 2014, pp. 1-6.

23. A. Mishra, M. Gupta and P. Sharma, "Enhancement of Underwater Images using Improved CLAHE," In 2018 IEEE International Conference on Advanced Computation and Telecommunication (ICACAT) December 2018, pp. 1-6.

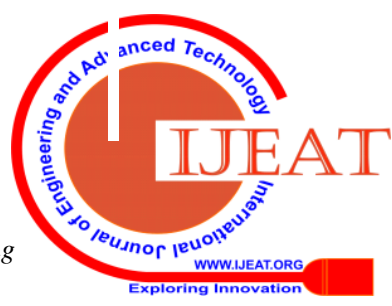


24. A. Thamizharasi, and J.S. Jayasudha,"An Illumination Invariant Face Recognition by Enhanced Contrast Limited Adaptive Histogram Equalization," ICTACT Journal on Image and Video Processing,vol.6, 2016.

25. H.B. Kekre and S.D. Thepade, "Improving Color to Gray and Back' using Kekre's luv color space,” In 2009 IEEE International Advance Computing Conference , March 2009, pp. 1218-1223.

26. H.B. Kekre and S.D. Thepade, "Color Traits Transfer to Grayscale Images,” In 2008 First International Conference on Emerging Trends in Engineering and Technology, July 2008, pp. 82-85.

27. S. Singh, M. Soni, A.K. Patel and R.S. Mishra,"Performance Evaluation of Spatial Domain Contrast Enhancement Techniques for Underwater Images," International Journal of Computer Applications, vol. 93, 2014.

28. V. Kumar and H. Bansal, "Performance evaluation of contrast enhancement techniques for digital images," International Journal of Computer Science and Technology, vol. 2, 2011, pp. 23-27.

29. A.K. Tripathi,S. Mukhopadhyay, and A.K. Dhara, "Performance Metrics for Image Contrast," In 2011 IEEE International Conference on Image Information Processing, November 2011, pp. 1-4.

30. H. Liang and D.S. Weller, Comparison-based image quality assessment for parameter selection, 2016, arXiv preprint arXiv:1601.04619.

\section{AUTHORS PROFILE}

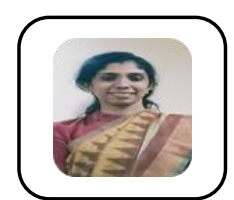

Deepa Abin is currently Assistant Professor in Computer Engineering Department at Pimpri Chinchwad College of Engineering affiliated to Savitribai Phule Pune University, Pune, Maharashtra, India. She is currently pursuing Ph.D. in Computer Engineering from PCCoE-SPPU. She has published several papers in International, National Conferences and Journals. Her domain of interest is Video Enhancement, Image Processing, Machine Learning and Artificial Intelligence.

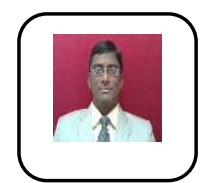

Sudeep D. Thepade is currently Professor in Computer Engineering Department at Pimpri Chinchwad College of Engineering affiliated to Savitribai Phule Pune University, Pune, Maharashtra, India. He has completed Ph.D. in 2011. He has more than 350 research papers to his credit published in International/ National Conferences and Journals. His domain of interest is Image Processing, Image Retrieval, Video Analysis, Video Visual Data Summarization, Biometrics and Biometric Liveness Detection. He is member of International Association of Engineers (IAENG) and International Association of Computer Science and Information Technology (IACSIT). He has served as Technical Program Committee member and Reviewer for Several International Conferences and Journals. 